S. Ishii

Nagoya Math. J.

Vol. 92 (1983), 39-50

\title{
CHOW INSTABILITY OF CERTAIN PROJECTIVE VARIETIES
}

\author{
SHIHOKO ISHII
}

\section{Introduction}

A pair $(X, D)$ of a projective variety $X$ and a very ample divisor $D$ on $X$ is called stable (resp. semi-stable, resp. unstable) if the Chow point corresponding to the embedding $\Phi_{|D|}: X \longrightarrow P^{N}$ is $S L(N+1)$-stable (resp. semi-stable, resp. unstable). The criterion for stability is one of the most important steps in proving the existence of moduli spaces. Up to these days, we know the following stable pairs $(X, D)$;

(1) $X$ is a non-singular curve of genus $g>0$ and $D$ is a very ample divisor of degree $>2 \mathrm{~g}$ (Mumford [4]).

(2) $X$ is a canonical surface and $D$ is $m K_{X}$ for sufficiently large $m$ (Gieseker [2]).

They imply the existence of the coarse moduli schemes of nonsingular curves of genus $>1$ and non-singular surfaces of general type, respectively.

On the other hand, we know that there are many divisors $D$ such that $(X, D)$ is unstable if $X$ has a "bad" singularity (cf. [4]). For nonsingular varieties, we did not know any example of unstable pairs $(X, D)$ except bundle-unstable ruled surfaces $X$ (Morrison [3]). The aim of this article is to give further examples of non-singular varieties which have many unstable divisors.

In Section 1, we obtain a criterion for a pair $(X, D)$ to be unstable in terms of cohomology and the intersection form of divisors.

Section 2 contains examples of unstable pairs $(X, D)$ satisfying the above criterion. We see that the blowing ups of projective cones with vertices of high multiplicity have many unstable divisors. Resolutions of "bad" singularities (cf. Propositions 8,10 ) have many unstable divisors. Moreover the blowing up of $\boldsymbol{P}^{n}(n \geq 2)$ at one point has many unstable divisors.

Received December 23, 1981.

Revised September 20, 1982. 
Throughout this paper, the base field $k$ is an algebraically closed field of arbitrary characteristic. Varieties are always irreducible reduced schemes of finite type over $k$. We will write $H^{i}(X, D)$ instead of the cohomology group $H^{i}(X, \mathcal{O}(D))$ for a Cartier divisor $D$. We refer the reader to [4] for the terminologies "stable", "semi-stable" etc. and the symbols

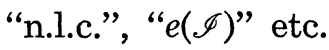

The author would like to thank Professors Y. Miyaoka and T. Oda for their useful suggestions and hearty encouragement.

\section{§1. A sufficient condition for instablity}

Fix a projective variety $X \subset \boldsymbol{P}^{N}$ of dimension $n>0$, homogeneous coordinates $X_{0}, \cdots, X_{N}$ on $\boldsymbol{P}^{N}$ and a 1-PS (one parameter subgroup) $\lambda$ of $S L(N+1)$. After changing the coordinates, we may assume;

$$
\lambda(t)=\left[\begin{array}{lll}
t^{\rho_{0}} & & 0 \\
& \ddots & \\
0 & & t^{\rho_{N}}
\end{array}\right] t^{-k},
$$

where $\rho_{0} \geq \rho_{1} \geq \cdots \geq \rho_{N}=0$ and $k=\sum_{i=0}^{N} \rho_{i} /(N+1)$. We define an ideal sheaf $\mathscr{I} \subset \mathcal{O}_{X \times A^{1}}$ in such a way that $\mathscr{I}\left(\mathcal{O}_{X}(1) \otimes \mathcal{O}_{A^{1}}\right)$ is the subsheaf generated by $\left\{t^{\rho_{i}} X_{i}\right\}, i=0, \cdots, N$.

Theorem 1 (Mumford [4], Theorem 2.9). An n-dimensional projective variety $X \subset \boldsymbol{P}^{N}$ is stable (resp. semi-stable) if and only if for any 1-PS $\lambda$ we have

$$
e(\mathscr{I})<\frac{(n+1) \operatorname{deg} X}{N+1} \sum_{i=1}^{N} \rho_{i} \quad(\text { resp. } \leq)
$$

For a sequence of integers $\rho_{0} \geq \rho_{1} \geq \cdots \geq \rho_{N}=0$, set $\mu_{i}=(N+1) \rho_{i}$. Then,

$$
\lambda(t)=\left[\begin{array}{lll}
t^{\mu_{0}} & & 0 \\
& \ddots & \\
0 & & t^{\mu_{N}}
\end{array}\right] t^{-\Sigma \rho_{i}}
$$

becomes a 1-PS of $S L(N+1)$. Since both sides of the quantities in Theorem 1 are linear in the $\rho_{i}$ 's jointly, we are always free to make such a change of scale, and henceforth shall do so without comment.

Let $X$ be a normal projective variety of dimension $n, H$ a Cartier divisor on $X$ and $E$ a subscheme of $X$ locally defined by a principal 
ideal (so, $E$ is considered as an effective Cartier divisor). Denote $\chi\left(X, \mathcal{O}_{X}(r H-s E)\right)$ by $h(r, s)$. Then by [5], $h(r, s)$ is a polynomial in $r$ and $s$ of the following form:

$$
\begin{aligned}
h(r, s)= & \sum_{i=0}^{n} \frac{\alpha_{i}}{(n-i) ! i !} r^{n-i} s^{i}+\sum_{i=0}^{n-1} \frac{\beta_{i}}{(n-1-i) ! i !} r^{n-1-i} s^{i} \\
& +(\text { lower order terms in } r, s) .
\end{aligned}
$$

For simplification of notation of the following discussions, we introduce the polynomials as follows;

$$
\begin{aligned}
A_{H E}(r, s)= & \left\{\left(-\sum_{i=0}^{n}\left(\begin{array}{c}
n+1 \\
i+1
\end{array}\right) \alpha_{i}\right) r^{n+1}+\sum_{i=0}^{n}\left(\begin{array}{c}
n+1 \\
i+1
\end{array}\right) \alpha_{i} r^{n-i} s^{i+1}\right\} h(r, s) \\
& +\left\{(n+1)\left(\sum_{i=0}^{n}\left(\begin{array}{c}
n \\
i
\end{array}\right) \alpha_{i} r^{n-i} s^{i}\right)\right\} \sum_{j=s+1}^{r} h(r, j),
\end{aligned}
$$

for integers $r, s$.

$$
\begin{aligned}
F_{H E}= & (n+1) \alpha_{0}\left\{\frac{1}{2} \sum_{i=1}^{n} \frac{\alpha_{i}}{(n-i) ! i !}+\sum_{i=1}^{n-1} \frac{\beta_{i}}{(n-1-i) !(i+1) !}\right\} \\
& -\frac{\beta_{0}}{(n-1) !} \sum_{i=1}^{n}\left(\begin{array}{c}
n+1 \\
i+1
\end{array}\right) \alpha_{i} .
\end{aligned}
$$

If $X$ is non-singular, then, by Riemann-Roch theorem, we can write $F_{H E}$ in terms of the intersection form:

$$
\begin{aligned}
F_{H, E}=\frac{(n+1)}{2}[ & H^{n}\left\{\frac{H^{n-1}(-E)}{n !}+\sum_{i=2}^{n} \frac{H^{n-i}(-E)^{i-2}\left(E^{2}+E K\right)}{(n-i) ! i !}\right\} \\
& \left.+n K H^{n-1}\left\{\sum_{i=1}^{n} \frac{H^{n-i}(-E)^{i}}{(n-i) !(i+1) !}\right\}\right]
\end{aligned}
$$

where $K$ is a canonical divisor on $X$.

Here, by easy but tedious calculation, we see that $F_{H, E}$ is the coefficient of the highest order term $r^{2 n}$ of $A_{H, E}(r, s)$, where we consider $A_{H, E}(r, s)$ as a polynomial in $r$.

Definition 1. Let $L$ be a line bundle of $X$ and $\lambda$ a 1-PS of $S L\left(h^{0}(X, L)\right)$. By $B_{\lambda}(L)$, we denote

$$
h^{0}(X, L) e(\mathscr{I})-(n+1) \operatorname{deg} L \sum_{i=0}^{h^{0}(X, L)-1} \rho_{i},
$$

under the notation of Theorem 1 . 
Note that $B_{\lambda}(L)>0$ for some $\lambda$ means instability of $(X, L)$, when $L$ is very ample.

Definition 2. Let $h$ and $h^{\prime}$ be integer valued functions defined on a subset $D$ of $Z^{a}(a \geq 1)$. If there exists $\varepsilon>0$ satisfying

$$
\left|h\left(m_{1}, \cdots, m_{a}\right)-h^{\prime}\left(m_{1}, \cdots, m_{a}\right)\right| \leq \varepsilon\left|\sum_{i=1}^{a} m_{i}\right|^{b} \quad(b \geq 0)
$$

for any $\left(m_{1}, \cdots, m_{a}\right) \in D$, we write $h \equiv h_{b}$ on $D$.

If there exist another function $h^{\prime \prime}$ defined on $D$ such that $h\left(m_{1}, \cdots, m_{a}\right)$ $\geq h^{\prime \prime}\left(m_{1}, \cdots, m_{a}\right)$ for all $\left(m_{1}, \cdots, m_{a}\right) \in D$ and $h^{\prime \prime} \underset{b}{\equiv} h^{\prime}$ on $D$, we write $h \geqq h^{\prime}$.

THEOREM 2. Let $f: X \rightarrow Y$ be the blowing up of a normal projectve variety $Y$ whose center is a subscheme $Z$ not necessarily irreducible or reduced. Let $E$ be a subscheme of $X$ locally defined by a principal ideal with support in $f^{-1}(Z)$. Put $H=f^{*} H_{0}$ where $H_{0}$ is an ample divisor on $Y$, and fix an integer $s \geq 0$. Let us assume the following:

(i) $h^{i}(X, r H-j E) \underset{n-2}{\equiv} 0$ on $\left\{(r, j) \in Z^{2} \mid s \leq j \leq r\right\}$ for any $i>0$, and

(ii) $F_{H, E}>0$.

Then we have the following conclusions.

(1) The case where the integer $s>0$ and $r H-s E$ is very ample for any large integer $r$.

(a) $(X, r H-s E)$ is unstable for sufficiently large $r$.

(b) $(X, r H-s E)$ is asymptotically unstable for sufficiently large $r$.

(2) The case where $s=0$.

(c) $\left(Y, H_{0}\right)$ is asymptotically unstable.

To prove the theorem, we give in the following lemma an evaluation of $B_{\lambda}$. This leads us a slightly more general criterion for instability. Unfortunately, we could not apply this lemma to finding any example of unstable pairs which are not covered by Theorem 2 .

Lemma 3. Let $H, E$ be Cartier divisors on $X$ and $E$ effective. Assume $h^{i}(X, r H-j E) \underset{n-2}{\equiv} 0$ on $\left\{(r, j) \in Z^{2} \mid 0 \leq j \leq r\right\}$ for any $i>0$. Then there exists a 1-PS $\lambda$ such that $B_{\lambda}(r H-s E) \underset{2 n-1}{\geqq} A_{H, E}(r, s)$ for $0 \leq s \leq r$.

Proof. Put $W_{i, j}=\Gamma(X, i H-j E)$ for $i, j \in Z$. Then $W_{r, s}$ has a filtra- 
tion $W_{r, s} \supset W_{r, s+1} \supset \cdots \supset W_{r, r}$. Let $X_{0}, X_{1}, \cdots, X_{N}$ be a basis of $W_{r, s}$ which is obtained by successive extension from a basis of $W_{r, r}$ according to the filtration. For this basis, define a 1-PS $\lambda$ by

$$
\lambda(t)=\left[\begin{array}{lll}
t^{\rho_{0}} & & \\
& \ddots & \\
& & \\
& & t^{\rho_{N}}
\end{array}\right],
$$

where $\rho_{i}=r-j$ if $X_{i} \in W_{r, j}$ and $X_{i} \notin W_{r, j+1}$. Then, $\Gamma\left(X \times A^{1}, \mathscr{I}\left(\mathcal{O}_{X}(r H-\right.\right.$ $\left.\left.s E) \otimes \mathcal{O}_{A^{1}}\right)\right)=$ the $k[t]$-submodule of $\Gamma\left(X \times A^{1}, \mathcal{O}_{X}(r H-s E) \otimes \mathcal{O}_{A^{1}}\right)$ generated by $W=t^{r-s} W_{r, s}+t^{r-s-1} W_{r, s+1}+\cdots+W_{r, r}$. Denote the $k[t]$-submodule of $W_{m r, m s} \otimes k[t]$ generated by the image of $W^{\otimes m}$ by $W^{m}$. Let $V^{m}$ be the $k[t]$-submodule of $W_{m r, m s} \otimes k[t]$ generated by $t^{m r-m s} W_{m r, m s}+\cdots+t W_{m r, m r-1}$ $+W_{m r, m r}$. Then $W^{m} \subset V^{m}$ in $W_{m r, m s} \otimes k[t]$. So we have

$$
\begin{array}{r}
\operatorname{dim}\left(W_{m r, m s} \otimes k[t] / W^{m}\right) \geq \operatorname{dim}\left(W_{m r, m s} \otimes k[t] / V^{m}\right) \\
\quad=(m r-m s) \operatorname{dim} W_{m r, m s}-\sum_{j=m s+1}^{m r} \operatorname{dim} W_{m r, j} .
\end{array}
$$

By Mumford [4, Proposition 2.6],

$$
\operatorname{dim} W_{m r, m s} \otimes k[t] / W^{m} \underset{n}{\equiv} \frac{e(\mathscr{I})}{(n+1) !} m^{n+1} .
$$

By the assumption of the theorem, $\operatorname{dim} W_{r, j} \underset{n-2}{\equiv} h(r, j)$. So we have

$$
\begin{aligned}
& (m r-m s) \operatorname{dim} W_{m r, m s}-\sum_{j=m s+1}^{m r} \operatorname{dim} W_{m r, j} \equiv(m r-m s) h(m r, m s) \\
& \quad-\sum_{j=m s+1}^{m r} h(m r, j) .
\end{aligned}
$$

By (3), (4) and (5),

$$
\begin{aligned}
& e(\mathscr{I}) \geq \text { n.l.c. }\{\left.(m r-m s) h(m r, m s)-\sum_{j=m s+1}^{m r} h(m r, j)\right\} \\
&=\text { n.l.c. }\left[\left\{(r-s) \sum_{i=0}^{n} \frac{\alpha_{i} r^{n-i} s^{i}}{(n-i) ! i !}-\sum_{i=0}^{n} \frac{\alpha_{i} r^{n+1}}{(n-i) !(i+1) !}\right.\right. \\
&\left.\left.\quad+\sum_{i=0}^{n} \frac{\alpha_{i} r^{n-i} s^{i+1}}{(n-i) !(i+1) !}\right\} m^{n+1}+(\text { lower order terms in } m)\right] \\
&=(n+1)(r-s) \sum_{i=0}^{n}\left(\begin{array}{c}
n \\
i
\end{array}\right) \alpha_{i} r^{n-i} s^{i}-\sum_{i=0}^{n}\left(\begin{array}{c}
n+1 \\
i+1
\end{array}\right) \alpha_{i} r^{n+1} \\
&+\sum_{i=0}^{n}\left(\begin{array}{c}
n+1 \\
i+1
\end{array}\right) \alpha_{i} r^{n-i} s^{i+1} .
\end{aligned}
$$


Since

$$
\begin{aligned}
& \operatorname{deg}(r H-s E)=\text { n.l.c. } h(m r, m s)=\sum_{i=0}^{n}\left(\begin{array}{c}
n \\
i
\end{array}\right) \alpha_{i} r^{n-i} s^{i}, \\
& h^{0}(r H-s E) \underset{n-2}{\equiv} h(r, s) \text { and } \sum \rho_{i} \equiv(r-s) h(r, s)-\sum_{n=1}^{r} h(r, j),
\end{aligned}
$$

the assertion which we want follows.

Q.E.D.

Proof of Theorem 2. Recall that $F_{H, E}$ is the coefficient of the highest order term $r^{2 n}$ of $A_{H, E}(r, s)$ which we consider as a polynomial in $r$. If $F_{H, E}>0$, we have $B_{\lambda}(r H-s E)>0$ for sufficiently large $r$, by Lemma 3 . Then (a) follows. The coefficient of the highest order term of $A_{H, E}(m r, m s)$ in $m$ is a polynomial in $r$ and $s$. This has $F_{H, E}$ as the coefficient of the highest order term $r^{2 n}$, when we consider it as a polynomial in $r$. By taking sufficiently large $r$ and $m$, we get $B_{\lambda}(m r H-m s E)>0$. Now (b) follows. By the normality of $Y$, we have $\Gamma(X, r H)=\Gamma\left(Y, r H_{0}\right)$. Take a filtration $\Gamma\left(Y, r H_{0}\right) \supset \Gamma(X, r H-E) \supset \cdots \supset \Gamma(X, r H-r E)$ on $\Gamma\left(Y, r H_{0}\right)$ and define a 1-PS $\lambda$ in the same way as the proof of Lemma 3. Now, we can take sufficiently large $r$ so that $B_{\lambda}(r H)$ is positive, since $F_{H, E}$ remains to be the coefficient of the highest order term of $A_{H, E}(r, 0)$ in $r$.

\section{$\S 2$. Chow instability of certain projective varieties}

In this section, we consider a blowing up $f: X \rightarrow Y$ of a normal variety of dimension $n \geq 2$ with center a subscheme $Z$ of dimension $d \leq n-2$ which is not necessarily irreducible nor reduced. Let $E$ be the scheme theoretic inverse image of the center $Z$ by the morphism $f$. This notation will be used throughout this section.

\section{(I) Blowing ups of projective cones}

Proposition 4. Let $V$ be a normal projective variety of dimension $n-1 \geq 1, L$ an ample divisor on $V$, and $Y_{t} \longrightarrow P^{N(t)+1}$ the projective cone over $\Phi_{|t L|}: V \longrightarrow P^{N(t)}$. Let $f_{t}: X_{t} \rightarrow Y_{t}$ be the blowing up with center the vertex. Put $\mathcal{O}_{Y_{t}}\left(H_{0 t}\right)=\left.\mathcal{O}_{P^{N(t)+1}}(1)\right|_{Y_{t}}, H_{t}=f^{*} H_{0 t}$ and $E_{t}=$ the fiber scheme of $f_{t}$ over the vertex.

Then, if we take a sufficiently large $t$, there exists an integer $r(s, t)>0$ for any $s>0$ so that the following hold.

(a) $\left(X_{t}, r H_{t}-s E_{t}\right)$ is unstable for any $r \geq r(s, t)$.

(b) $\left(X_{t}, r H_{t}-s E_{t}\right)$ is asymptotically unstable for any $r \geq r(s, t)$.

(c) $\left(Y_{t}, H_{0 t}\right)$ is asymptotically unstable. 
Proof of Proposition 4. Put

$$
\chi(V, m L)=\left(a_{0} /(n-1) !\right) m^{n-1}+\left(a_{1} /(n-2) !\right) m^{n-2}+\cdots+a_{n-1} .
$$

Note that $a_{0}>0$. Take a large integer $t$ in such a way that

$$
\begin{aligned}
& n a_{0} t>2 a_{1}, \\
& H^{i}(V, m t L)=0 \quad \text { for any } m>0 \text { and } i>0 .
\end{aligned}
$$

In the following, we will omit the suffix $t$ for simplicity of notations.

First we show $H^{i}(X, r H-j E)=0$ for any $i>0$ and any $j, r$ such that $1 \leq j \leq r$, and $h^{i}(X, r H) \underset{n-2}{\equiv} 0$ for any $i>0$ and $r>0$. Let $p: X \rightarrow V$ be the projection of the canonical $\boldsymbol{P}^{1}$-bundle structure. Then, $H=p^{*}(t L)$ $+E$. So by the property (2), we get

$$
H^{i}(X, r H-r E)=H^{i}(V, r t L)=0
$$

for any $i>0, r>0$. The exact sequences of sheaves

$$
\begin{gathered}
0 \longrightarrow \mathcal{O}_{X}(r H-(j+1) E) \longrightarrow \mathcal{O}_{X}(r H-j E) \longrightarrow \mathcal{O}_{E}(-j E) \longrightarrow 0 \\
\mathcal{O}_{V}(j t L)
\end{gathered}
$$

$(0 \leq j \leq r-1)$ induce the exact sequences of cohomology groups

$$
H^{i}(X, r H-(j+1) E) \longrightarrow H^{i}(X, r H-j E) \longrightarrow H^{i}(V, j t L)
$$

for $0 \leq j \leq r-1$. Here, by (2) and (3), we get $H^{i}(X, r H-j E)=0$ for $1 \leq j \leq r$ as desired. We also see that $h^{i}(X, r H)=h^{i}\left(V, \mathcal{O}_{V}\right)$ for any $r>0$ and $i>0$.

Next we will show $F_{H, E}>0$. Since $\chi(X, r H-s E)=\chi\left(X, p^{*}(r t L)\right)=$ $\chi(V, r t L)$, we can easily show that $h(r, s)=\chi(X, r H-s E)=\sum_{j=s}^{r} \chi(V, j t L)$. The right hand side is

$$
\begin{aligned}
& \left(\frac{a_{0} t^{n-1}}{n !}\right)\left(r^{n}-s^{n}\right)+\frac{a_{0} t^{n-1}+2 a_{1} t^{n-2}}{2(n-1) !} r^{n-1}+\frac{a_{0} t^{n-1}-2 a_{1} t^{n-2}}{2(n-1) !} s^{n-1} \\
& \quad+(\text { lower order terms in } r, s) .
\end{aligned}
$$

So we have

$$
\begin{aligned}
& \alpha_{0}=a_{0} t^{n-1}, \quad \alpha_{i}=0(1 \leq i \leq n-1), \quad \alpha_{n}=-a_{0} t^{n-1}, \\
& \beta_{0}=\frac{1}{2}\left(a_{0} t^{n-1}+2 a_{1} t^{n-2}\right), \quad \beta_{i}=0(1 \leq i \leq n-2) \\
& \beta_{n-1}=\frac{1}{2}\left(a_{0} t^{n-1}-2 a_{1} t^{n-2}\right) .
\end{aligned}
$$


Substitute them into $F_{H, E}$, then we have

$$
\begin{aligned}
F_{H, E} & =(n+1) \alpha_{0}\left(\frac{1}{2} \frac{\alpha_{n}}{n !}+\frac{\beta_{n-1}}{n !}\right)-\frac{\beta_{0} \alpha_{n}}{(n-1) !} \\
& =\frac{a_{0} t^{2 n-3}}{2 n !}\left(n a_{0} t-2 a_{1}\right),
\end{aligned}
$$

which is positive by (1).

Proposition 5. In the notation of Proposition 4, let $L$ be a very ample divisor of $V$ such that $\Phi_{|L|}: V \longrightarrow P^{n}$ is a hypersurface of degree $d$ with $2 \leq d \leq n+1$.

Then the results (a), (b) and (c) hold for any $t \geq 1$.

Proof. By the proof of Proposition 4, we have only to show (1) and (2) for any $t \geq 1$.

For (1), use $a_{0}=d, a_{1}=(n+1-d) d / 2$. Then,

$$
n a_{0} t-2 a_{1}=d(d-1+(t-1) n)>0 \quad \text { for any } t \geq 1 .
$$

The condition (2) is easily checked.

Example 6. The projective cone over a non-singular conic in $\boldsymbol{P}^{2}$ and a non-singular quadratic surface in $\boldsymbol{P}^{3}$ satisfies the condition of Proposition 5. So the results (a), (b) and (c) hold for any $t \geq 1$.

\section{(II) Resolutions of singularities}

In the rest of this section, we assume $X$ is non-singular.

Proposition 7. Assume $X$ is non-singular. Put $H=f^{*} H_{0}$, where $H_{0}$ is an ample divisor on $Y$. Then,

$$
\begin{aligned}
F_{H, E}=\frac{(n+1)}{2}[ & H^{n}\left\{\sum_{i=n-d}^{n} \frac{H^{n-i}(-E)^{i-2}\left(E^{2}+E K\right)}{(n-i) ! i !}\right\} \\
& \left.+n K H^{n-1}\left\{\sum_{i=n-d}^{n} \frac{H^{n-i}(-E)^{i}}{(n-i) !(i+1) !}\right\}\right],
\end{aligned}
$$

where $K$ is the canonical divisor on $X$.

Proof. Since $X$ is non-singular, we have $\alpha_{i}=H^{n-i}(-E)^{i}$ and $\beta_{i}=$ $(-1 / 2) K H^{n-1-i}(-E)^{i}$. Here, note that $\alpha_{i}=0, \beta_{i-1}=0$ for $n-i>d$, since $f$ is the blowing up of a $d$-dimensional subscheme.

Q.E.D.

Definition 3. Let $G_{H, E}=H^{d}(-E)^{n-d-2}\left(E^{2}+E K\right)$. We say $E$ is of 
general type (resp. elliptic type, rational type) for $H_{0}$ if $G_{H, E}>0$ (resp. $\left.G_{H, E}=0, G_{H, E}<0\right)$.

Note that if $f$ is a blowing up with center a zero dimensional subscheme, then the above types are independent of $H_{0}$. Especially, if $X, Y$ are surfaces, $G_{H, E}=2 g-2$, where $g$ is the vertual genus of $E$.

Proposition 8. Let $E$ be of general type for $H_{0}$. By replacing $H_{0}, H$ by $t H_{0}, t H$, respectively, for a sufficiently large integer $t$, we get the following.

(a) $(X, r H-s E)$ is unstable for sufficiently large $r$, for any fixed $s>0$.

(b) $(X, r H-s E)$ is asymptotically unstable for sufficiently large $r$, for any fixed $s>0$.

(c) $\left(Y, H_{0}\right)$ is asymptotically unstable.

Proof. The positive $G_{H, E}$ is the coefficient of the highest order term in $t$ of $F_{t H, E}$. So $F_{t H, E}>0$ for sufficiently large $t$ (condition (ii) of Theorem 2).

If we show $h^{i}(X, r t H-j E) \underset{n-2}{\equiv} 0$ on $\left\{(j, r) \in Z^{2} \mid 0 \leq j \leq r\right\}$ for $i>0$, then the proposition follows by Theorem 2 .

First we claim that if we choose sufficiently large $t$, there exists an integer $k>0$ such that $H^{i}(X, r t H-j E)=0$ for $i>0$ and any $j, r$ with $k \leq j \leq r$. In fact take a sufficiently large integer $t$ such that $t H-E$ is ample on $X$ and $t H_{0}$ is very ample on $Y$. We can take a sequence $X=$ $D_{0} \supset D_{1} \supset \cdots \supset D_{h}(h \geq 2)$ of subschemes of $X$ such that $D_{i}$ is of pure $(n-i)$-dimension, the defining ideal of $D_{i}$ in $D_{i-1}$ is isomorphic to $\mathcal{O}_{D_{i-1}}(-t H)$ and $\mathcal{O}_{D_{h}}(t H)=\mathcal{O}_{D_{h}}([6]$, Lemma 1$)$. Since $t H=f^{*} t H_{0}$ and $t H_{0}$ is very ample, $D_{h}$ must be in $f^{-1}(A)$, where $A$ is a 0 -dimensional subscheme of $Y$. We have the exact sequences

$$
\begin{aligned}
H^{i}\left(D_{j},\left.k(t H-E)\right|_{D_{j}}\right) & \longrightarrow H^{i}\left(D_{j},(k+1) t H-\left.k E\right|_{D_{j}}\right) \\
& \longrightarrow H^{i}\left(D_{j+1},(k+1) t H-\left.k E\right|_{D_{j+1}}\right)
\end{aligned}
$$

for $0 \leq j \leq h-1, i>0$ and $k>0$. By the choice of $t$, there is an integer $k_{0}>0$ such that $H^{i}\left(D_{j},\left.k(t H-E)\right|_{D_{j}}\right)=0$ for $i>0,0 \leq j \leq h$ and any $k \geq k_{0}$. Since $-E$ is relatively ample for $f$, we may also assume that $H^{i}\left(D_{h},-\left.k E\right|_{D_{h}}\right)=0$ for $i>0$ and any $k \geq k_{0}$. Substitute them into the above exact sequences, then we have $H^{i}\left(D_{j},(k+1) t H-\left.s E\right|_{D_{j}}\right)=0$ for $i>0,0 \leq j \leq h$. Thus we show the case $r=k+1$. By induction on $r$, consequently we have $H_{i}^{i}\left(D_{j}, r t H-\left.k E\right|_{D_{j}}\right)=0$ for any $r, k$ with $k_{0} \leq k \leq$ $r, 0 \leq j \leq h$ and $i>0$. 
Next we show that, if we fix $j$ such as $0 \leq j \leq k_{0}-1, h^{i}(X, r t H-j E)$ $\underset{n-2}{\equiv} 0$ as a function of $r$ for $i>0$. This will complete the proof of the proposition. Fix an integer $j$ such as $0 \leq j \leq k_{0}-1$. Since $H_{0}$ is ample on $Y$, there exists an integer $r(j)$ such that $H^{p}\left(Y, \mathcal{O}\left(r t H_{0}\right) \otimes R^{q} f_{*} \mathcal{O}(-j E)\right)$ $=0$ for $p>0, q \geq 0$ and any $r \geq r(j)$. Consider the spectral sequence,

$$
E_{2}^{p, q}=H^{p}\left(Y, \mathcal{O}\left(r t H_{0}\right) \otimes R^{q} f_{*} \mathcal{O}(-j E)\right) \Longrightarrow E^{p+q}=H^{p+q}(X, r t H-j E) .
$$

Since $E_{2}^{p, q}=0$ for $p \neq 0$, we have

$$
H^{0}\left(Y, \mathcal{O}\left(r t H_{0}\right) \otimes R^{i} f_{*} \mathcal{O}(-j E)\right) \simeq H^{i}(X, r t H-j E)
$$

for $i>0$ and $r \geq r(j)$. Since the support of $\mathcal{O}\left(r t H_{0}\right) \otimes R^{i} f_{*} \mathcal{O}(-j E)$ is of dimension $\leq n-2, h^{i}(X, r t H-j E) \underset{n-2}{\equiv} 0$ as a function of $r$ Q.E.D.

For a non-singular canonically polarized surface $X$ (i.e. whose canonical divisor is ample), $\left(X, K_{X}\right)$ is asymptotically stable by Gieseker [2]. On the other hand, $X$ may have an unstable divisor. In fact, we have an example of a non-singular canonical polarized surface which has many unstable divisors.

EXAMPLE 9 (canonically polarized variety with many unstable divisors). Let $Y \subset \boldsymbol{P}^{n+1}$ be an irreducible reduced hypersurface of degree $d$ with only one isolated singular point $(1,0, \cdots, 0)$. Assume the defining polynomial $F\left(X_{0}, \cdots, X_{n+1}\right)$ is of the form $F_{m} X_{0}^{d-m}+F_{m+1} X_{0}^{d-m-1}+\cdots+F_{d}$, where $F_{i}$ is a homogeneous polynomial in $X_{1}, \ldots, X_{n+1}$ of degree $i, m$ is an integer with $2 \leq m \leq d-3$ and the hypersurface $Y_{m} \subset \boldsymbol{P}^{n}$ defined by $F_{m}=0$ is non-singular.

Then the blowing up $f: X \rightarrow Y$ with center $(1,0, \cdots, 0)$ is a resolution of singularity. If $m \geq n+2$, then $d \geq n+5$. So, $X$ is a canonically polarized variety and $E$ is of general type for any ample divisor $H_{0}$ on $Y$, since $E$ is isomorphic to $Y_{m}$ whose canonical divisor is very ample. Applying Proposition 8, we get instabilities (a), (b) and (c).

Next, we consider a special case of elliptic type.

Proposition 10. Assume $\mathcal{O}_{X}\left(E+K_{X}\right) \otimes \mathcal{O}_{E} \simeq \mathcal{O}_{E}$ and $H^{n-1} K_{X}<0$. Then the assertions (a), (b) and (c) in Proposition 8 hold.

Proof. By the assumption,

$$
F_{H, E}=\left(\frac{1}{2}\right) n(n+1)\left(H^{n-1} K_{X}\right)\left(\sum_{i=n-d}^{n} H^{n-i}(-E)^{i} /(n-i) !(i+1) !\right) .
$$


We see that $n(n+1)\left(H^{n-1} K_{X}\right)\left(H^{d}(-E)^{n-d} / d !(n-d+1) !\right)$ is the coefficient of the highest order term of $F_{t H, E}$ in $t$. By $H^{d}(-E)^{n-d}<0$ and the assumption, the coefficient as above is positive. We are done in the same way as in Proposition 8.

ExAmPLe 11. Let $C$ be an elliptic curve and $X$ be a ruled surface defined by a decomposable vector bundle $\mathcal{O} \oplus \mathscr{L}$ on $C$, where $\mathscr{L}$ is an ample line bundle. Then the contraction $f: X \rightarrow Y$ of the minimal section $E$ satisfies the conditions of the above proposition.

Next, from the above, we get another example of unstable pair of elliptic type, which is not the blowing up of a projective cone with the vertex as center. Let $X^{\prime}$ be the blowing up of $X$ with center a point on $E$. Then the contraction $f^{\prime}: X^{\prime} \rightarrow Y^{\prime}$ of the proper transform $E^{\prime}$ of $E$ satisfies the conditions of Proposition 10.

For the rational type, we already had examples which have many unstable divisors (cf. Example 6). Here, we mention another example of rational type which is also the blowing up of a projective cone with the vertex as center.

Example 12. Let $f: X \rightarrow \boldsymbol{P}^{n}$ be the blowing up of $\boldsymbol{P}^{n}(n \geq 2)$ with a point as center. $H_{0}$ be a hyperplane in $\boldsymbol{P}^{n}$, and $H$ be $f^{*} H_{0}$. Let $E$ be the exceptional divisor.

Then,

(a) $(X, r H-s E)$ is unstable for sufficiently large $r$, for any fixed $s>0$,

(b) $(X, r H-s E)$ is asymptotically unstable for any $r, s$ with $r>s$ $>0$, and

(c) $\left(\boldsymbol{P}^{n}, r H_{0}\right)$ is semi-stable for any $r \geq 2$.

The result (c) was given by Kempf [1]. For (a) and (b), we note that $F_{H, E}=0$. We get the conclusions by direct calculations of $A_{H, E}(r, s)$.

\section{REFERENCES}

[1] Kempf, G., Instability in invariant theory, Ann. of Math., 108 (1978), 299-316.

[ 2 ] Gieseker, D., Global moduli for surfaces of general type, Invent. Math., 43 (1980), 269-304.

[ 3 ] Morrison, I., Projective stability of ruled surfaces, Invent. Math., 56 (1980), 269-304.

[4] Mumford, D., Stability of projective varieties, L'Enseignement Math., XXIII, (1977), 39-110.

[5] Snapper, E., Polynomials associated with divisors, J. Math. Mech., 9 (1960), 123139. 
[6] Ishii, S., Some projective contraction theorems, Manuscripta Math., 22 (1977), 343-358.

Department of Mathematics Tokyo Metropolitan University Fukazawa-2, Setagaya

Tokyo 158 Japan 